\title{
Weak gravitational lensing by compact objects in fourth order gravity
}

\author{
Zsolt Horváth* and László Á. Gergely \\ Departments of Theoretical and Experimental Physics, \\ University of Szeged, Dóm tér 9, Szeged 6720, Hungary \\ David Hobil \\ Department of Physics and Astronomy, University of Calgary, Calgary Alberta T2N 1N4, Canada

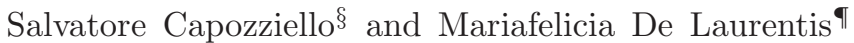 \\ Dipartimento di Scienze Fisiche, Universit'a di Napoli "Federico II", I-80126, Napoli, Italy \\ INFN Sez. di Napoli, Compl. Univ. di Monte S. Angelo, Edificio G, Via Cinthia, I-80126, Napoli, Italy

\begin{abstract}
We discuss weak lensing characteristics in the gravitational field of a compact object in the lowenergy approximation of fourth order $f(R)$ gravity theory. The particular solution is characterized by a gravitational strength parameter $\sigma$ and a distance scale $r_{c}$ much larger than the Schwarzschild radius. Above $r_{c}$ gravity is strengthened and as a consequence weak lensing features are modified compared to the Schwarzschild case. We find a critical impact parameter (depending upon $r_{c}$ ) for which the behavior of the deflection angle changes. Using the Virbhadra-Ellis lens equation we improve the computation of the image positions, Einstein ring radii, magnification factors and the magnification ratio. We demonstrate that the magnification ratio as function of image separation obeys a power-law depending on the parameter $\sigma$, with a double degeneracy. No $\sigma \neq 0$ value gives the same power as the one characterizing Schwarzschild black holes. As the magnification ratio and the image separation are the lensing quantities most conveniently determined by direct measurements, future lensing surveys will be able to constrain the parameter $\sigma$ based on this prediction.
\end{abstract}

\section{INTRODUCTION}

The recent advent of the so-called "Precision Cosmology" along with galactic observations indicate that General Relativity (GR) with standard matter sources disagrees with an increasing number of observational data, e. g. those coming from IA-type Supernovae, used as standard candles, large scale structure ranging from galaxies up to superclusters [1 3], and galactic rotation curves. In addition, from a theoretical point of view, being not renormalizable, GR fails to be quantized in any standard way (see [4]). Therefore at the extreme ultra-violet and infrared scales GR is not and cannot be the definitive theory of Gravitation despite the fact that it successfully addresses a wide range of phenomena and the Newtonian weak field limit is correctly recovered.

In order to interpret the recent observational data in the framework of GR, the introduction of unknown dark matter (DM) (to address dynamical phenomena as the formation of self-gravitating astrophysical structures) and dark energy (DE) (to address the problem of cosmic acceleration) seems to be necessary: however, the price of preserving the simplicity of the Hilbert-Einstein Lagrangian has been the introduction of rather odd-behaving physical entities that, up to now, have not been revealed by any experiment at fundamental scales. This situation has led to several attempts devoted either to recover the validity of GR at any scale, or to construct alternative gravity theories that suitably generalize the Einsteinian one. The philosophy of these two schemes is that in the former case one has to modify the matter sector introducing DM and DE, in the latter approach the dynamics of the geometry (i.e. the left-hand-side of the Einstein equations) is modified but with the constraint to recover GR at local scales.

Higher-order theories of gravity (both in metric [5 9] and Palatini [10 12] formulations) represent an interesting approach able to fruitfully cope with both dark matter and dark energy problems. A further approach is based on scalar - tensor theories of gravity but it can be shown that higher-order theories and scalar tensor ones can be related by conformal transformations (see, e.g., [6, 13] and references therein).

It has been widely demonstrated that such theories can agree with the cosmological observations of the Hubble flow

\footnotetext{
*Electronic address: zshorvath@titan.physx.u-szeged.hu

$\dagger^{\dagger}$ Electronic address: gergely@physx.u-szeged.hu

‡Electronic address: hobill@ucalgary.ca

$\S$ Electronic address: capozzie@na.infn.it

TElectronic address: felicia@na.infn.it
} 
[14, 15] and the large scale structure evolution [16]. In addition, in the weak field limit, the gravitational potential turns out to be modified [17 20] in such a way that interesting consequences to galactic dynamics may be achieved without violating, at the same time, the constraints on the parametrized post-Newtonian (PPN) parameters coming from Solar System tests [21].

If alternative theories of gravitation are able to explain both cosmological and local observations without the introduction of exotic energy-momentum sources, then one might ask how would the differences between these alternative theories be compared to GR with the unusual sources. It is proposed that gravitational lensing might be able to act a means for determining which theory governs the gravitational interaction, through measurements of image separations and the brightnesses of those multiple images.

It is well known that the deflection of light observed during the Solar eclipse of 1919 was one of the first experimental confirmations of Einsteinian GR. Gravitational lensing, i.e. the deflection of light rays crossing the gravitational field of a compact object referred to as the lens, has become one of the most astonishing successes of GR and it represents nowadays a powerful tool capable of putting constraints on the dynamics of gravitational structures at different scales, from stars to galaxies and clusters of galaxies, and from the large scale structure to cosmological parameters 22, 23]. If we modify the Lagrangian of the gravitational field, it is obvious that gravitational lensing should be affected. It is therefore mandatory to investigate how gravitational lensing behaves in the framework of alternative theories of gravity to develop a further check on the existence of DM and DE.

In particular, one has to verify that the phenomenology of standard gravitational lensing is recovered in the limit as the modified theory of gravity reduces to GR, since several observations point to the validity of GR. However, it is worth stressing that the presence of DM has to be invoked in such cases, in particular for large-scale structure (see e.g. the case of Bullet Cluster [24]). On the other hand, it is worth exploring whether deviations from the classical results for the main lensing quantities could be detected and act as clear signatures for modified theories of gravity. A preliminary study in this direction is in 25] where the gravitational lensing, in the Newtonian limit and in the Jordan frame, for a generic $f\left(R, R_{\mu \nu}^{\mu \nu}, R_{\mu \nu \alpha \beta} R^{\mu \nu \alpha \beta}\right)$ is considered. In this paper, the modifications of the Hilbert-Einstein action are induced by corrections to the Newtonian potential due to the Riemann tensor.

As a first step towards such an ambitious task we consider here power-law fourth order theories, i.e. we replace the Ricci scalar $R$ in the gravity Lagrangian with the function $f(R) \propto R^{n}$ (with $n$ a positive integer) and investigate how this affects the gravitational lensing in the theory. In Ref. [26] the gravitational lensing in $f(R)$ theories with a Yukawa-type correction in the potential was investigated and the conclusion reached was that weak lensing could not discern between these theories and general relativity. We revisit this issue by using better lens equations together with a post-Newtonian approximation, both of which can account for higher order deviations away from general relativity. We discuss weak lensing due to compact objects of the $R^{n}$ theory, which have been already used to analyze rotation curves of galaxies in [27] and in [28].

The paper is organized as follows. The next section provides a short summary of $f(R)$ theories, focusing on the special $R^{n}$ case and we review the post-Newtonian solution representing the compact object. The modifications from GR can be interpreted as an effective energy-momentum tensor. We discuss the referring energy conditions in the Appendix. We discuss in Section III how the predictions of weak gravitational lensing are different in the fourth order theory and in general relativity. For this we determine the image locations, Einstein ring radii, magnification factors and the flux ratio, for various model parameters. We then demonstrate that the flux ratio as function of image separation has a different power-law dependence for each model parameter. We summarize our findings in the Concluding Remarks.

\section{A SPHERICALLY SYMMETRIC COMPACT OBJECT IN $f(R)$-GRAVITY}

\section{A. Generalities}

Let us consider now a particular class of higher order theories of gravity, the $f(R)$-gravity theories, that are the most natural extension of GR where the Hilbert-Einstein action is modified with a general function of the scalar curvature [5, 6]. A general action is

$$
\mathcal{A}=\int d^{4} x \sqrt{-g}\left[f(R)+\mathcal{L}_{m}\right]
$$


where $f(R)$ is a generic function of the Ricci curvature scalar $R$, differentiable at least up to the second order, $g$ is the determinant of the metric and $\mathcal{L}_{m}$ is the standard matter Lagrangian. ${ }^{1}$ The general analysis of ghosts of $\mathrm{f}(\mathrm{R})$ gravity can be found in Ref. 31]. Varying the action with respect to the metric components $g_{\mu \nu}$, one obtains the generalized field equations that can be recast as [9, 32]

$$
G_{\mu \nu}=\frac{1}{f^{\prime}(R)}\left\{\frac{1}{2} g_{\mu \nu}\left[f(R)-R f^{\prime}(R)\right]+f^{\prime}(R)_{; \mu \nu}-g_{\mu \nu} \square f^{\prime}(R)\right\}+\frac{T_{\mu \nu}^{(m)}}{f^{\prime}(R)}
$$

where $G_{\mu \nu}=R_{\mu \nu}-\frac{R}{2} g_{\mu \nu}$ and $T_{\mu \nu}^{(m)}$ are the Einstein tensor and the standard matter stress - energy tensor, respectively. The prime denotes derivative with respect to $R$. The two terms $f^{\prime}(R)_{; \mu \nu}$ and $\square f^{\prime}(R)$ imply fourth order derivatives of the metric $g_{\mu \nu}$ so that these models are also referred to as fourth order gravity. Starting from Eq. (2) and adopting the Robertson-Walker metric, it is possible to show that the Friedmann equations may still be written in the usual form provided that an effective curvature fluid (hence the name of curvature quintessence) is added to the matter term with energy density and pressure depending on the choice of $f(R)[9]$.

\section{B. Power-law models with spherical symmetry}

In the absence of matter, when spherical symmetry holds, the trace of the field equations (2),

$$
3 \square f^{\prime}(R)+R f^{\prime}(R)-2 f(R)=0,
$$

combined with the 00 - component leads to [ $[$ ]

$$
f^{\prime}(R)\left(3 \frac{R_{00}}{g_{00}}-R\right)+\frac{1}{2} f(R)-3 \frac{\nabla_{0} \nabla_{0} f^{\prime}(R)}{g_{00}}=0 .
$$

Equation (4) is completely general and holds for any function $f(R)$. Note that even if the metric is stationary so that $\partial_{0} g_{\mu \nu}=0$, due to the existence of non-zero Christoffel symbols entering the second covariant derivative, $f^{\prime}(R)_{; 00}=\frac{1}{2} g^{i j} \partial_{i} g_{00} \partial_{j} f^{\prime}(R)$ is non-vanishing.

The simplest choice for $f(R)$ is a power-law like $f(R) \propto R^{n}$ with $n$ the slope of the Lagrangian (clearly, with $n=1$, we recover the Einstein theory).

In a spherically symmetric setup whenever $R$ is vanishing, constant, or depending only on the radial coordinate $r$ (hence the space-time is stationary), the Jebsen-Birkhoff theorem holds, and the space-time is also static [6], [33]. Therefore we search for spherically symmetric solutions of Eqs. (2). In general, we can write the space-time metric as :

$$
d s^{2}=-A(r) d t^{2}+B(r) d r^{2}+r^{2} d \Omega^{2}
$$

where $d \Omega^{2}=d \theta^{2}+\sin ^{2} \theta d \varphi^{2}$ is the line element on the unit sphere. Eq. (4) reduces to

$$
R_{00}(r)=\frac{2 n-1}{6 n} A(r) R(r)-\frac{n-1}{2 B(r)} \frac{d A(r)}{d r} \frac{d \ln |R(r)|}{d r},
$$

while the trace equation reads:

$$
\square R^{n-1}(r)=\frac{2-n}{3 n} R^{n}(r)
$$

Note that as soon as $n=1$, Eq. (7) reduces to $R=0$, which, when inserted into Eq. (6), gives $R_{00}=0$ and then the standard Schwarzschild solution is recovered. In general, expressing $R_{00}$ and $R$ in terms of the metric (5), Eqs. (6) and (7) become a system of two nonlinear coupled differential equations for the two functions $A(r)$ and $B(r)$.

\footnotetext{
${ }^{1}$ It is possible to take into account also the Palatini approach in which the metric $g$ and the connection $\Gamma$ are considered independent fields (see for example [29]). Here we consider the Levi-Civita connection and use the metric approach. See [5, 30] for a detailed comparison between the two pictures.
} 


\section{A low-energy, far-field solution}

We present here a solution of the low-energy approximation of the field equations, following Ref. [27].

A physically motivated hypothesis is to search for (Schwarzschild-like) solutions of the form

$$
A(r)=\frac{1}{B(r)}=1+\frac{2 \Phi(r)}{c^{2}},
$$

where $\Phi(r)$ is the gravitational potential at the distance $r$ from a pointlike mass $m$. Further, deviations from the Newtonian $1 / r$ potential are introduced as

$$
\Phi\left(r ; \sigma, r_{c}\right)=-\frac{G m}{2 r}\left[1+\left(\frac{r}{r_{c}}\right)^{\sigma}\right],
$$

with a strength parameter $\sigma$ and a characteristic distance $r_{c}$. It is straightforward to see that, for $\sigma=0$, the Newtonian potential is recovered and the metric reduces to the Schwarzschild one (GR-case). The cases $\sigma>1$ (as the correction to the Newtonian potential asymptotically diverges) and $\sigma=1$ (as the correction is a constant, obstructing asymptotic flatness) are excluded.

With $\Phi(r)$ inserted into Eqs. (6) and (7), they both reduce to algebraic equations, which lead to relations between $\sigma$ and $n$ :

$$
(n-1)(\sigma-3)\left[-\sigma(1+\sigma) V_{1} \eta^{\sigma-3}\right]^{n-1}\left[\mathcal{P}_{1}+\frac{\sigma}{\eta} V_{1} \mathcal{P}_{0}\right]=0 .
$$

Here $\eta=r / r_{c}, V_{1}=G m / c^{2} r_{c}$ and

$$
\begin{gathered}
\mathcal{P}_{0}=3(\sigma-3)^{2} n^{3}-\left(5 \sigma^{2}-31 \sigma+48\right) n^{2}-\left(3 \sigma^{2}-16 \sigma+17\right) n-\left(\sigma^{2}-4 \sigma-5\right), \\
\mathcal{P}_{1}=3(\sigma-3)^{2}(1-\sigma) n^{3}+(\sigma-3)^{2}(5 \sigma-7) n^{2}-\left(3 \sigma^{3}-17 \sigma^{2}+34 \sigma-36\right) n+\left(\sigma^{2}-3 \sigma-4\right) \sigma .
\end{gathered}
$$

Eq. (10) is identically satisfied for particular values of $n$ and $\sigma$. It should be noted that when deriving Eq. (6) from Eq. (4), we assumed $R \neq 0$, which eliminates the case $n=1$. Secondly, $\sigma=3$ may also be rejected since as noted above, all $\sigma>1$ lead to divergences. The third factor cannot vanish as it would imply either $\sigma=0$ or $\sigma=-1$ for which $n$ is less than unity. Then we have to solve for the last factor. For large scale-lengths $r_{c}$ the parameter $V_{1} \ll 1$ and acts as a post-Newtonian parameter, while both $n$ and $\sigma$ are of order unity, hence $\mathcal{P}_{0}$ and $\mathcal{P}_{1}$ are comparable. Therefore in the post-Newtonian regime we can approximate the last factor with

$$
\mathcal{P}_{1}(n, \sigma) \eta=0,
$$

which is an algebraic equation for $\sigma$ as function of $n$, allowing for the following three solutions:

$$
\sigma=\left\{\begin{array}{l}
\frac{3 n-4}{n-1} \\
\frac{12 n^{2}-7 n-1-\sqrt{p(n)}}{q(n)} \\
\frac{12 n^{2}-7 n-1+\sqrt{p(n)}}{q(n)}
\end{array}\right.
$$

with :

$$
p(n)=36 n^{4}+12 n^{3}-83 n^{2}+50 n+1
$$

and

$$
q(n)=6 n^{2}-4 n+2 .
$$

It is easy check that, for $n=1$ the second solution (14) gives $\sigma=0$, which reduces to the Newtonian potential. The other two solutions do not fulfill this limit, hence we keep the second solution obtaining the low-energy, far-field expression

$$
\sigma=\frac{12 n^{2}-7 n-1-\sqrt{36 n^{4}+12 n^{3}-83 n^{2}+50 n+1}}{6 n^{2}-4 n+2}
$$




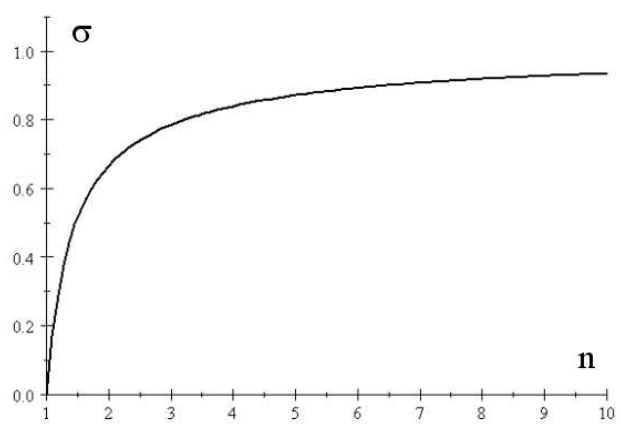

FIG. 1: The dependence of correction parameter $\sigma$ in the potential upon the exponent $n$.

The dependence of $\sigma$ upon the exponent $n$ is represented in Fig. 1

In the case $n=1$ (implying $\sigma=0$ ) the potential reduces to the Newtonian $\Phi_{N}$, as expected. The potential also reduces to the Newtonian value at $r=r_{c}$. For smaller values of $r$ gravity is weakened compared to the Newtonian values, while for $r>r_{c}$ gravity is strengthened.

\section{Interpretation of the parameters}

While the power $\sigma$ of the correction term is a universal quantity (since it depends on the exponent $n$ entering the gravity Lagrangian), the scale-length $r_{c}$ is related to the integration constants that have to be set to solve the fourth order differential equations of the theory. The radius $r_{c}$ can be considered as a further gravitational radius complementing the Schwarzschild radius, originating in the fact that we consider a fourth order theory (compared to the second order GR) and as such it introduces two further degrees of freedom of the gravitational field. We expect $r_{c}$ to be related to the peculiarities of each gravitational system. Therefore it can take different values depending upon the system's mass and typical length scale.

The fact that gravity is strengthened above $r_{c}$ is illustrated in Fig. 2 by plotting the ratio of the potentials $\Phi\left(r ; \sigma, r_{c}\right) / \Phi_{N}(r)$ as function of $\sigma$ and $r$ for the ranges $0 \leq \sigma<1$ and $r \geq r_{c}$ with a suitably chosen value of $r_{c}$. In the case of a typical spiral galaxy we identify $r_{c}$ with the bulge radius $r_{b u l g e}$, in order to have the Newtonian potential at $r \approx r_{\text {bulge }}$, cf. Eq. (9). We have chosen a typical bulge radius 3240 parsec (pc), corresponding to the order $10^{20} \mathrm{~m}$ [34], and typical bulge mass of $10^{10} M_{\odot}$. Therefore gravity is strengthened outside $r_{\text {bulge }}$ as compared to the Newtonian case, providing an alternative to dark matter as a source for a flat rotation curve [35]. We note also that the ratio $r_{c} / r_{S}$ is of order $10^{6}$.

The energy conditions for the effective energy momentum tensor are investigated in the Appendix.

\section{WEAK LENSING IN FOURTH ORDER GRAVITY}

\section{A. The deflection angle}

In Ref. [32] the weak lensing by point-like massive objects characterized by the potential (9) was investigated, where a deflection angle

$$
\delta=\frac{2 G m}{c^{2} b}\left[1+\frac{\sqrt{\pi}(1-\sigma) \Gamma(1-\sigma / 2)}{2 \Gamma(3 / 2-\sigma / 2)}\left(\frac{b}{r_{c}}\right)^{\sigma}\right]
$$

was derived. Here $b=|\theta| D_{l}$ is the impact parameter (defined as the distance of the lensing object to the straight line trajectory, which would occur in the absence of the lensing object). For $\sigma=0$ the deflection angle reproduces the Schwarzschild value $\delta_{S}=4 G \mathrm{~m} / \mathrm{c}^{2} b$, while in the limit $\sigma \rightarrow 1$ the deflection angle is one half of $\delta_{S}$.

In order to investigate the behavior of $\delta$ in between the limiting values, we represent the deflection angle as function of $\sigma$ in Fig. 3. Three conclusions stand out: i) by increasing the impact parameter at any fixed value of $\sigma$, the deflection angle always decreases, as in the Schwarzschild case; ii) there is a critical value of the ratio $b / r_{c}$ at $\left(b / r_{c}\right)_{c r i t}=2$, below which the deflection angle monotonically decreases with increasing $\sigma$, and above which there is a single maximum at 


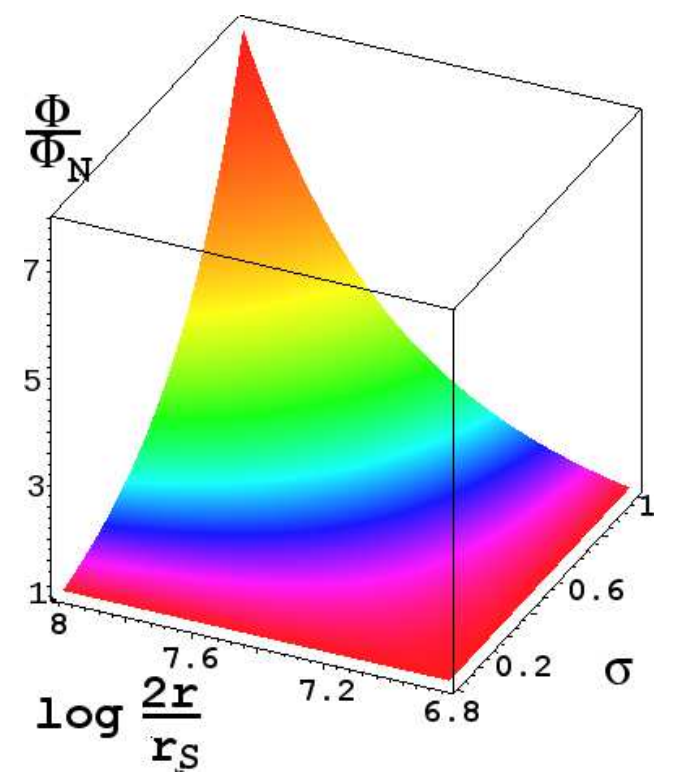

FIG. 2: The ratio of the power-law $f(R)$ gravitational potential and the Newtonian gravitational potential as a function of $\sigma \in[0,1)$ and of the logarithm of the distance $r \geq r_{c}$, normalized to $r_{S}$ (the Schwarzschild radius of the lens). We have chosen a typical bulge mass of $m_{\text {bulge }} \approx 10^{10} M_{\odot}$ and typical bulge radius $r_{c} \approx r_{\text {bulge }} \approx 3240$ pc.

some $\sigma_{\delta_{\max }}$; iii) the parameter value $\sigma_{\delta_{\max }}$ increases with the value of $b / r_{c}$. The rate of decrease of $\delta$ with increasing $b$ is lessened as compared to the Schwarzschild case for small impact parameters of order $r_{c}$.

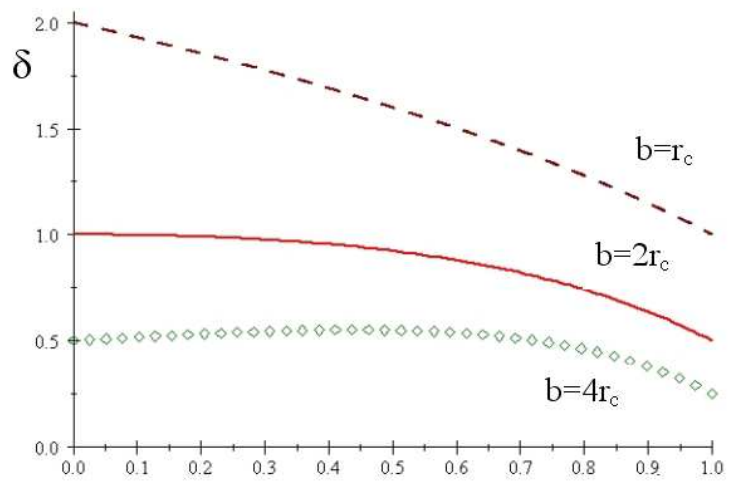

a)

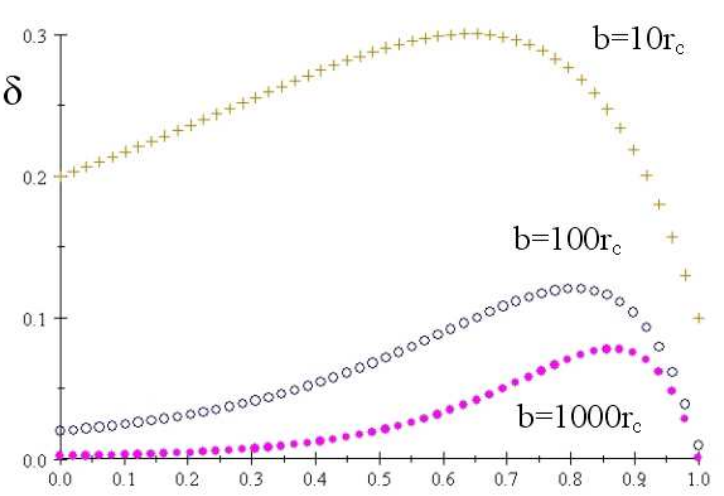

b) $\sigma$

FIG. 3: The plots show the $\delta(\sigma)$ dependence in radians for different values of $b / r_{c}$, in units $2 G m / c^{2} r_{c}=1$, with the choice $r_{c}=3240 \mathrm{pc}$. The respective values of $b / r_{c}$ are from top to bottom 1 (dashed), 2 (solid), 4 (diamond) on panel (a) and 10 (cross), 100 (circle), 1000 (dotted) on panel (b). The critical behavior appears at $b / r_{c}=2$.

\section{B. The lensing geometry}

The lensing geometry is shown on Fig. 4. The optical axis $O L N$ is defined by the observer position $O$, the lens position $L$, and intersects the source plane at $N$. In the source plane $S$ represents the location of the source and $I_{1,2}$ the locations of the two images. We use the notations $D_{l s}$ and $D_{l}$ for the projection of the lens-source and observer-lens distances onto the optical axis respectively [36]. The observer-source distance is $D_{s}=D_{l}+D_{l s}$. The source is located at an angle $\beta$ from the optical axis, chosen to be "above" the optical axis. Images are located at angles $\theta_{1,2}$ with respect to the optical axis and they can be either positive (for the image above the optical axis) or 


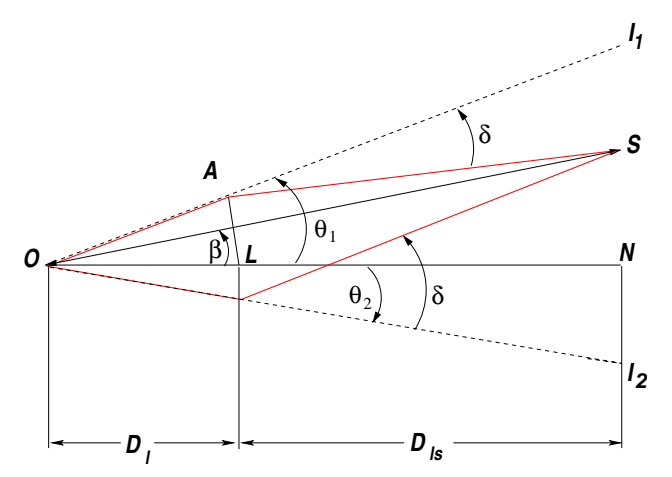

FIG. 4: The lensing geometry (see text for discussion of the symbols). Positive angles are represented with counterclockwise directed arcs.

negative (for the image below the optical axis). For either of the images we denote $s=\operatorname{sgn} \theta$, such that $|\theta|=s \theta$. We follow the convention that the deflection angle is $\delta>0$ whenever the light is bent towards the optical axis, cf. Ref. [38]. Similarly as in Ref. [36], we characterize the mass by the dimensionless parameter $\bar{\varepsilon}=G m / c^{2} L$, with $L=D_{s} D_{l} / D_{l s}$.

In Ref. [32] the leading order lens equation

$$
|\theta|-s \beta-\frac{D_{l s}}{D_{s}} \delta=0
$$

was employed for the discussion of the weak lensing. Many authors have obtained more accurate results for weak lensing by using explicit trigonometric relationships. One of the most useful is that given by Virbhadra and Ellis [39], where their lens equation is

$$
\tan |\theta|-\tan (s \beta)-\frac{D_{l s}}{D_{s}}[\tan |\theta|+\tan (\delta-|\theta|)]=0 .
$$

Alternatively even more general lens equations have been derived in Ref. [40]:

$$
\sin \alpha=\frac{D_{l s}}{D_{s}} \cos |\theta| \cos \left[\arcsin \left(\frac{D_{s}}{D_{l s}} \sin (|\theta|-\alpha)\right)\right][\tan |\theta|+\tan (\delta-|\theta|)],
$$

where $\alpha:=\theta-\beta$ is the "reduced" deflection angle, or in Ref. [36]:

$$
\frac{2 D_{l}}{D_{s}} \cos \left(\frac{\delta}{2}-|\theta|\right) \cos |\theta| \sin \frac{\delta}{2}+\cos (\delta-|\theta|)(\sin |\theta|-s \cos |\theta| \tan \beta)-\sin \delta=0 .
$$

Which expression to use in the subsequent calculations will depend on the ability of current technology to resolve the differences between the predictions made by the various proposed lens equations. Assuming that the observations of the lensed image positions are (or soon will be) capable of resolving angular differences on the order of microarcseconds (see e.g. Ref. [41]) one can compare the accuracy of the different lens equations and then choose the simplest expression that is compatible with the available astrometric precision.

The left panel of Fig. 5. compares the lens equations (18) and (17), while right panel compares the lens equations (19) and (18). The difference between the Einstein angles $\theta_{V E}$, obtained from Eq. (18) and $\theta_{0}$, obtained from Eq. (17), are represented as function of the parameters $\sigma$ and $\bar{\varepsilon}$ on the left panel of Fig. [5 and they are of order of microarcseconds. The differences between the Einstein angles $\theta_{V E}$ and $\theta_{D S}$, obtained from Eq. (19) are shown on the right panel of Fig. 5, and they are of order of nanoarcseconds. Remarkably the two figures are identical in shape, but differing in 3 orders of magnitude. A comparison between Eqs. (20) and (18) leads to a similar conclusion.

Hence Fig. 5. illustrates that both Eqs. (18) and (19) lead to identical corrections of Eq. (17) in the lensing behavior within less than $1 \%$. In what follows we employ the simpler lens equation (18), rather than (19) or the second order lens equation derived in Ref. [36], as it provides sufficient accuracy for confrontation with observations.

\section{Image positions and magnifications}

In Ref. [32] the image positions, the radius of the Einstein ring, image magnifications and the Paczynski curve in microlensing experiments were also estimated. In this follow-up paper we improve on the accuracy of the weak lensing 

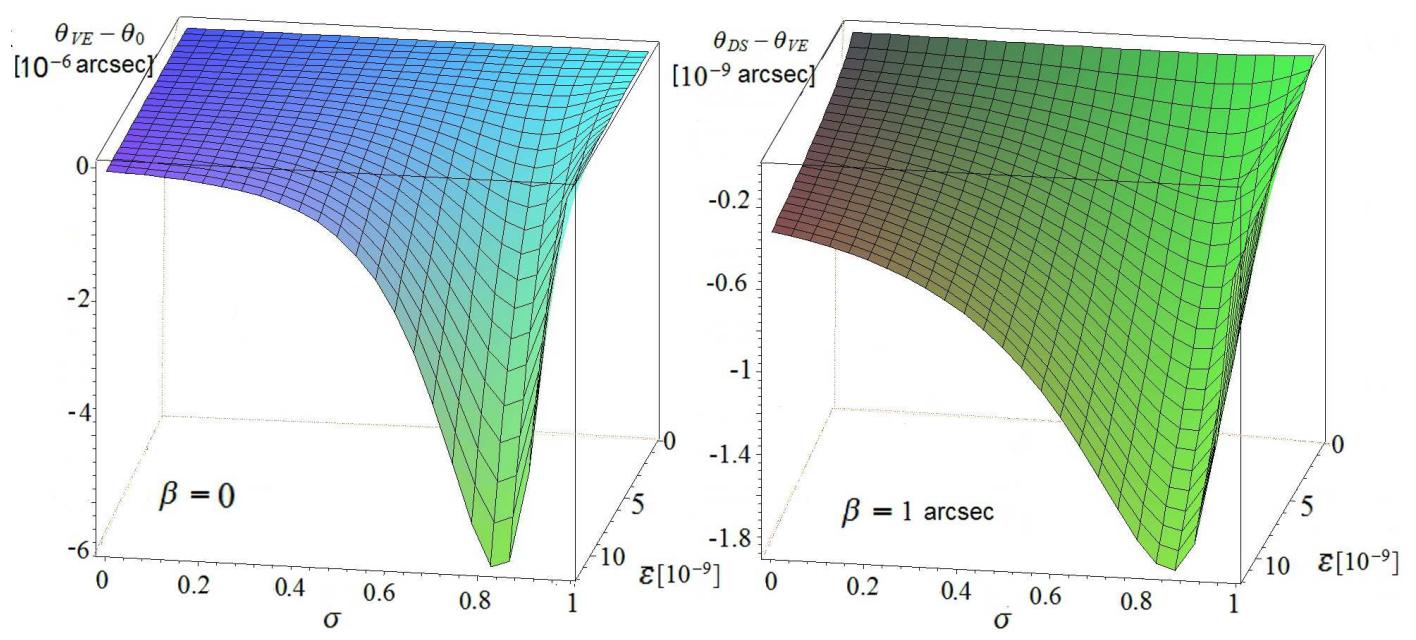

FIG. 5: (a) The difference between the Einstein angles $\theta_{V E}$, obtained from Eq. (18) and $\theta_{0}$, obtained from Eq. (17), as function of the parameters $\sigma$ and $\bar{\varepsilon}$. (b) The difference between the positive apparent angles $\theta_{V E}$ and $\theta_{D S}$ [obtained from Eq. (19)] of the images of a source located at $\beta=1$ arcsec. While the two surfaces have the same shape, it should be noted that the vertical scales differ by 3 orders of magnitude. We set the distances $D_{l}=1 \mathrm{Mpc}$ and $D_{l s}=2 \mathrm{Mpc}$.

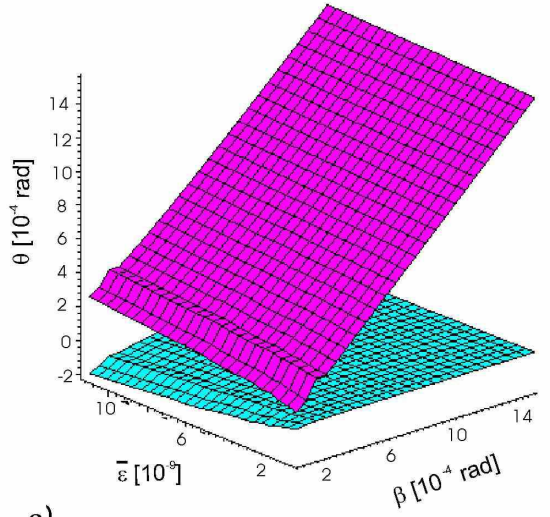

a)

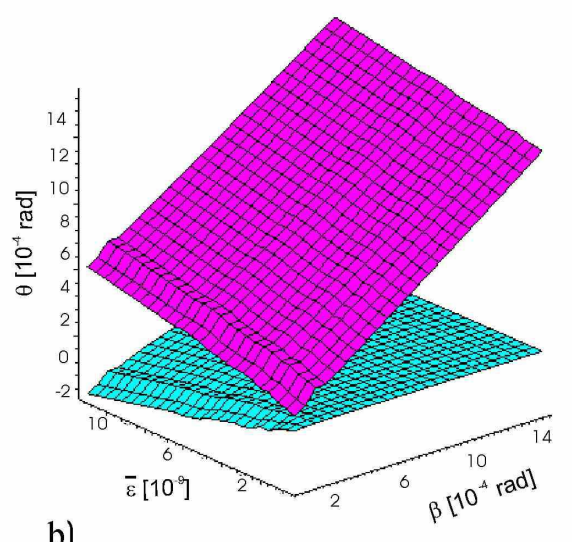

b)

FIG. 6: The image positions $\theta$ as function of $\bar{\varepsilon}$ and $\beta$ for $\sigma=0.25$ (a) and $\sigma=0.75$ (b), for the distances $D_{l}=1$ Mpc and $D_{l s}=2 \mathrm{Mpc}$. The angle $\beta$ is varied up to $0.0015 \mathrm{rad}$, similarly as on Fig $4(\mathrm{~b})$ of Ref. 36. With decreasing $\beta$, the image separations shrink accordingly. At $\beta=0$ the angle $\theta$ represents the angular radius of the Einstein ring. As we expect the $\beta=0$ sections of the surfaces are symmetric with respect to the plane $\theta=0$.

characteristics, by employing a more accurate lens equation with the focus on the behavior of dimensionless observable quantities which can be derived from the image positions and magnification factors. Our task is to determine the measurable differences between the predictions of the fourth order theory and those of GR.

A weak lens equation was derived in Ref. [36] exclusively by trigonometric considerations and was applied to the computations of the image positions, magnifications and flux ratios to second order accuracy (both in the mass-related and tidal charge related small parameters) for brane-world black holes. In addition it was shown, how the VirbhadraEllis lens equation follows as an approximation (agreement is reached in the first order of the perturbations). For the purposes of the present paper, it is recognized that second order and higher effects will not be measurable by current telescope technology, and therefore we will utilize the Virbhadra-Ellis lens equation [38], 39], [41], [42], together with 
the deflection angle (16) derived in Ref. [32]. This generalizes the approach of Ref. [32], where the leading order lens equation [Eq. (12) there] was employed for the discussion of weak lensing effects.

The numerical solution of the system of equations (16) and (18) gives the positions of the images as function of $\bar{\varepsilon}$ and $\beta$, represented on Fig. [6 for $\sigma=0.25$ and 0.75 , respectively. In both cases decreasing $\beta$ decreases the image separations. As expected, the $\beta=0$ sections give symmetric curves with respect to the planes $\theta=0$. This is because at $\beta=0$ the angle $\theta=\theta_{E}$ represents the angular radius of the Einstein ring. For small impact parameter (implying small $\beta$ ) the image separations obey $\left(\theta_{1}-\theta_{2}\right)_{\sigma=0.25}<\left(\theta_{1}-\theta_{2}\right)_{\sigma=0.75}$, whereas for large values of $\beta$ the image separations behave as $\left(\theta_{1}-\theta_{2}\right)_{\sigma=0.25}>\left(\theta_{1}-\theta_{2}\right)_{\sigma=0.75}$. This indicates that our analysis based on the Virbhadra-Ellis lens equation is more accurate than the first post-Newtonian order calculation performed in Section VIII. in Ref. [43], which states that $f(R)$ gravity is indistinguishable from general relativity and is consistent with the observational value of the post-Newtonian parameter $\gamma=1+(2.1 \pm 2.3) \times 10^{-5}$.

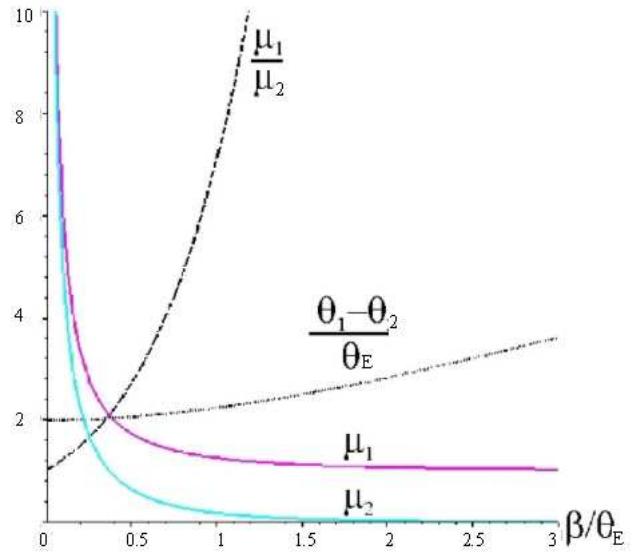

a)

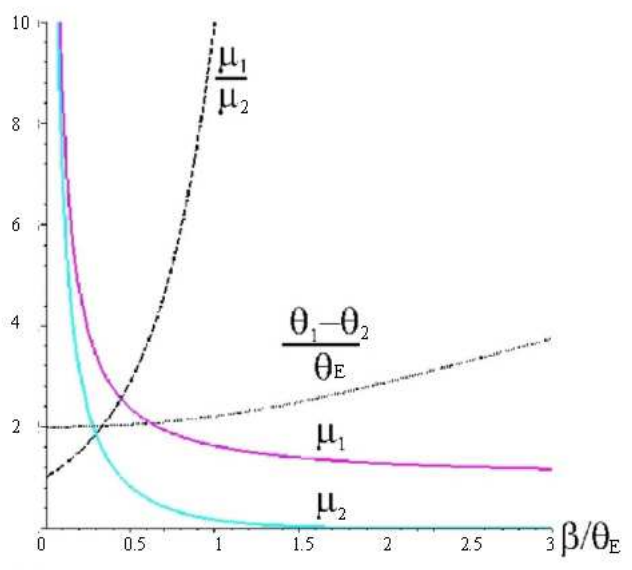

b)

FIG. 7: The image separations and magnifications as functions of $\beta / \theta_{E}$ for $\sigma=0.25$ (a) and $\sigma=0.75$ (b). We fixed $\bar{\varepsilon}=3.375 \times 10^{-9}$, while the distances $D_{l}=1 \mathrm{Mpc}$ and $D_{l s}=2 \mathrm{Mpc}$ were chosen for the plots. The upper and lower solid curves represent the primary and secondary image magnification factors, respectively; their ratio is the dashed curve; and the dotted curve is the normalized image separation.

The magnification of the images are defined as

$$
\mu_{1,2}=\left|\frac{d \theta_{1,2}}{d \beta} \frac{\theta_{1,2}}{\beta}\right| .
$$

For Schwarzschild lensing $\mu_{1}-\mu_{2}=1$ always holds. Figure 7 shows the image separations and magnifications as functions of $\beta / \theta_{E}$ for $\sigma=0.25$ and 0.75 . The upper and lower solid curves represent the primary and secondary image magnification factors, respectively; their ratio is the dashed curve; and the dotted curve is the image separation normalized with respect to the Einstein angle. The strongest effect appears on the ratio of magnifications, which increases more rapidly with $\beta$ as $\sigma$ increases. The reason for this is that the primary image is magnified more for larger values of $\sigma$.

\section{Power-law behavior}

In a lensing observation the most straightforward measurements are 1) the angular separation between the two images and 2) the ratio of the magnification factors. The first measurement does not require information on the location of the lens position, needed to define the individual image positions. The second measurement does not require an absolute measure of image brightness, since we are taking a ratio between the two.

Therefore we plot the ratio of the magnification factor for the primary and secondary images as function of the image separation (normalized to the Einstein angle), on the $\log -\log$ scale, in Fig. 8 . The $\sigma=0$ curve characterizes the lensing by a Schwarzschild black hole, the colored curves correspond to the fourth order gravity lensing for the parameter values of $\sigma$ ranging from 0.1 to 0.9999 . 

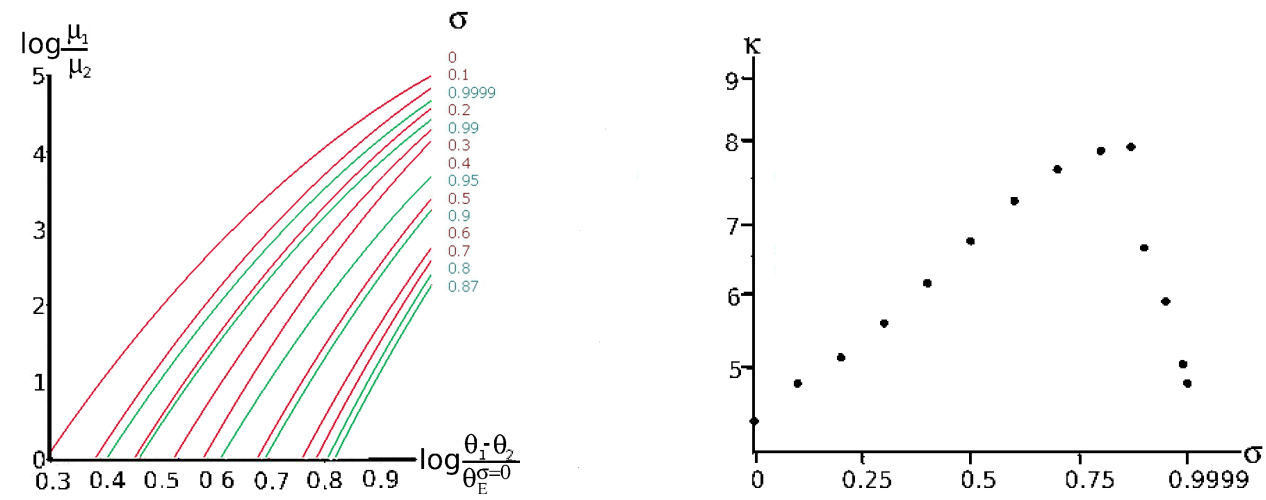

FIG. 8: The ratio of the magnification factor of the primary and secondary images as function of the image separation (normalized to the Einstein angle $\theta_{E}^{\sigma=0}$, characterizing the $\sigma=0$ case), on log-log scale for a series of $\sigma$ values (left panel). There is a double degeneracy in $\sigma$ of the power $\kappa$, represented as a function of $\sigma$ (right panel). The plot refers to $\bar{\varepsilon}=3.375 \times 10^{-9}$ and distances $D_{l}=1 \mathrm{Mpc}, D_{l s}=2 \mathrm{Mpc}$.

TABLE I: The exponents $\kappa$ associated with the power law scaling of the magnification ratio as function of image separation for various values of $\sigma$, up to a double degeneracy.

\begin{tabular}{|l||c|c|c|c|c|c|c|c|c|c|c|c|c|c|}
\hline$\sigma$ & 0 & 0.1 & 0.2 & 0.3 & 0.4 & 0.5 & 0.6 & 0.7 & 0.8 & 0.87 & 0.9 & 0.95 & 0.99 & 0.9999 \\
\hline$\kappa$ & 4.39 & 4.87 & 5.21 & 5.64 & 6.15 & 6.69 & 7.20 & 7.60 & 7.84 & 7.92 & 6.60 & 5.92 & 5.12 & 4.87 \\
\hline
\end{tabular}

Similar to Ref. [36], it is found that for image separations greater than about 2.5 times the Einstein angle, the ratio of the magnification factors for each value of $\sigma$ obeys a power-law relationship

$$
\frac{\mu_{1}}{\mu_{2}} \propto\left(\frac{\Delta \theta}{\theta_{E}}\right)^{\kappa}
$$

The different slopes $\kappa$ of the curves indicate power-law behaviors with different exponents, which are presented in Table 1 and in Fig. 8 .

Given a large enough number of measurements of image separations and image brightnesses, these power-law relations provide an observational signature that can distinguish among the fourth order $f(R)$ theories with different $\sigma($ or $n)$.

\section{CONCLUDING REMARKS}

In this paper we have analyzed the weak lensing signatures of a fourth order $\left[f(R)=R^{n}\right]$ gravity compact object, with gravitational potential given in the post-Newtonian regime by Eqs. (9) and (15). This introduces a new parameter $\sigma \in[0,1)$, which governs the deviation from the Newtonian gravitational potential for different values of $n$. General relativity is contained as the special case $n=1$ (corresponding to the model parameter $\sigma=0$ ). For any other value of the parameter $n$ (or $\sigma$ ) the gravitational attraction increases at distances larger than $r_{c}$ as compared to the prediction of Newtonian gravity.

The lensing properties of such compact objects were analyzed before in Ref. [32], based on the small angle lens equation [42]. In this paper we have improved upon this approach, by employing the first order accurate VirbhadraEllis lens equation (18), or with equivalent results the Dábrowski-Schunck lens equation (19).

We analyzed the dependences upon $\sigma$ and upon the impact parameter $b$ of the deflection angle (16). The deflection angle decreases with increasing impact parameter for all $\sigma$. There is a transition at a critical value $\left(b / r_{c}\right)_{c r i t}=2$, below which the deflection angle monotonically decreases with increasing $\sigma$, and above which there is a single maximum. This maximum value increases with the impact parameter.

The image positions as a function of the lensing mass and source position, also the image magnifications and their ratio as function of source position show features similar to those in the Schwarzschild case. Nevertheless, in contrast with previous claims in the literature [25], 26] these lensing quantities depend upon $\sigma$.

We have computed the image positions for two values of $\sigma$. For the larger value of $\sigma$, the image separation grows faster with an increase in the mass and grows more slowly as the source moves away from the optical axis. 
For the same source position the magnification factors of the images increase with $\sigma$, especially the one for the primary image. The increases in their ratio $\mu_{1} / \mu_{2}$ is even more significant.

Using the most easily measurable lensing observables, the ratio of the magnifications is shown to have a powerlaw dependence on the image separations, with the power strongly depending on $\sigma$. The power is the smallest for Schwarzschild black holes $(\sigma=0)$, then it increases with $\sigma$ to a critical value, after which it decreases again. This behavior provides a means for future gravitational lensing observations to either establish the value of $\sigma$ up to a double degeneracy or falsify the power-law gravitational potential discussed in this paper if $\sigma=0$ is confirmed. Given that the next generation of radio telescopes will easily be able to resolve images to less than milliarcsecond (in fact tens of microarcseconds) accuracy, the different rates at which the ratio of the magnifications changes should be able to provide a significant observational signature constraining the validity of $f(R)$ gravitational theories.

\section{Acknowledgements}

Z. H. and L. Á. G. were supported by the European Union and European Social Fund Grants TÁMOP 4.2.2/B10/1-2010-0012 and TÁMOP-4.2.2.A-11/1/KONV-2012-0060, by COST Action MP0905 "Black Holes in a Violent Universe", and by the Hungarian Scientific Research Fund (OTKA) Grant No. 81364. D. H. acknowledges support from an NSERC Discovery Grant.

\section{Appendix A: Energy conditions in the low-energy, far-field regime}

The low-energy, far-field solution (5), (8)-(9) and (15) holds for vacuum, hence there are no energy conditions to observe in the $f(R)$ theory.

The first term in Eq. (2) however can be interpreted as an effective energy-momentum tensor of geometric origin within the context of General Relativity. In terms of the metric decomposition

$$
g_{\mu \nu}=-u_{\mu} u_{\nu}+n_{\mu} n_{\nu}+h_{\mu \nu},
$$

[with $h_{\mu \nu}$ the metric on the 2 -sphere surfaces, $u^{\mu}=(1 / \sqrt{A}, 0,0,0)$ the temporal unit vector and $n^{\mu}=(0, \sqrt{A}, 0,0)$ the radial unit vector], the energy-momentum tensor of the spherically symmetric, static curvature fluid is decomposed as

$$
T_{\mu \nu}=\rho u_{\mu} u_{\nu}+p_{r} n_{\mu} n_{\nu}+p_{t} h_{\mu \nu}
$$

or

$$
T_{\mu \nu}=\left(\rho+p_{t}\right) u_{\mu} u_{\nu}+\left(p_{r}-p_{t}\right) n_{\mu} n_{\nu}+p_{t} g_{\mu \nu}
$$

Here $\rho$ is the energy density, $p_{r}$ and $p_{t}$ the the radial and tangential pressures, respectively:

$$
\begin{aligned}
\rho & =T_{\mu \nu} u^{\mu} u^{\nu}=T_{00}, \\
p_{r} & =T_{\mu \nu} n^{\mu} n^{\nu}=T_{11}, \\
p_{t} & =\frac{1}{2}\left(T_{\mu \nu} h^{\mu \nu}\right)=\frac{T_{22}}{r^{2}} .
\end{aligned}
$$

The equivalent energy-momentum tensor for the fourth order theory reads:

$$
T_{\mu \nu}^{e q u i v}=(1-n)\left\{\frac{1}{2 n} g_{\mu \nu} R+\left(g_{\mu}^{\rho} g_{\nu}^{\sigma}-g_{\mu \nu} g^{\rho \sigma}\right)\left[(1-n) \nabla_{\rho}(\ln |R|) \nabla_{\sigma}(\ln |R|)-\nabla_{\rho} \nabla_{\sigma}(\ln |R|)\right]\right\}
$$

vanishing, as required, in the GR case $n=1$. Here the curvature scalar is given by

$$
\begin{aligned}
R & =\frac{2(A-1)}{r^{2}}+\frac{4 A_{, r}}{r}+A_{, r r} \\
& =-\sigma(\sigma+1) \frac{G m}{c^{2} r_{c}^{\sigma}} r^{\sigma-3},
\end{aligned}
$$


while

$$
\begin{aligned}
\nabla_{\sigma} \ln |R| & =\frac{(\sigma-3)}{r} \delta_{\sigma}^{1}, \\
\nabla_{\rho} \nabla_{\sigma}(\ln |R|) & =-\frac{(\sigma-3)}{r^{2}} \delta_{\rho}^{1} \delta_{\sigma}^{1}-\frac{(\sigma-3)}{r} \Gamma_{\rho \sigma}^{1},
\end{aligned}
$$

where the required Christoffel symbols are

$$
\begin{aligned}
\Gamma_{00}^{1} & =-\Gamma_{11}^{1}=\frac{A A_{, r}}{2}=\frac{G m}{2 c^{2} r^{2}}\left[1+(1-\sigma)\left(\frac{r}{r_{c}}\right)^{\sigma}\right]\left(1-\frac{G m}{c^{2} r}\left[1+\left(\frac{r}{r_{c}}\right)^{\sigma}\right]\right) \\
& \approx \frac{G m}{2 c^{2} r^{2}}\left[1+(1-\sigma)\left(\frac{r}{r_{c}}\right)^{\sigma}\right], \\
\Gamma_{22}^{1} & =\frac{\Gamma_{33}^{1}}{\sin ^{2} \theta}=-r A=-r\left(1-\frac{G m}{c^{2} r}\left[1+\left(\frac{r}{r_{c}}\right)^{\sigma}\right]\right) .
\end{aligned}
$$

The $\approx$ denotes expansion to first order in the post-Newtonian parameter $G m / c^{2} r$.

The equivalent energy-momentum tensor becomes

$$
\begin{aligned}
T_{\mu \nu}^{e q u i v}= & (1-n) g_{\mu \nu}\left\{-\frac{\sigma(\sigma+1) G m}{2 n c^{2} r_{c}^{\sigma}} r^{\sigma-3}+\frac{(\sigma-3)}{r^{2}}\left[[1+(1-n)(\sigma-3)] A+r g^{\rho \sigma} \Gamma_{\rho \sigma}^{1}\right]\right\} \\
& +(1-n) \frac{(\sigma-3)}{r^{2}}\left\{[(1-n)(\sigma-3)+1] g_{\mu}^{1} g_{\nu}^{1}+r \Gamma_{\mu \nu}^{1}\right\}
\end{aligned}
$$

From Eqs. A4 and the intermediary result

$$
\begin{aligned}
g^{\rho \sigma} \Gamma_{\rho \sigma}^{1}= & \frac{\left(1-A^{2}\right) A_{, r}}{2}+2 r^{-1} A \\
= & \frac{1}{4 r}\left(\frac{G m}{c^{2} r}\right)^{2}\left[1+\left(\frac{r}{r_{c}}\right)^{\sigma}\right]\left[1+(1-\sigma)\left(\frac{r}{r_{c}}\right)^{\sigma}\right]\left(1-\frac{G m}{4 c^{2} r}\left[1+\left(\frac{r}{r_{c}}\right)^{\sigma}\right]\right) \\
& +2 r^{-1}\left\{1-\frac{G m}{2 c^{2} r}\left[1+\left(\frac{r}{r_{c}}\right)^{\sigma}\right]\right\} \\
\approx & 2 r^{-1}\left\{1-\frac{G m}{2 c^{2} r}\left[1+\left(\frac{r}{r_{c}}\right)^{\sigma}\right]\right\}
\end{aligned}
$$

we obtain the first order post-Newtonian expressions

$$
\begin{aligned}
\rho & =\frac{n-1}{r^{2}}\left(\rho_{r}^{0}-\frac{G m}{c^{2} r} \rho_{r}^{1}\right), \\
p_{r} & =\frac{1-n}{r^{2}}\left(p_{r}^{0}-\frac{G m}{c^{2} r} p_{r}^{1}\right), \\
p_{t} & =\frac{1-n}{r^{2}}\left(p_{t}^{0}-\frac{G m}{c^{2} r} p_{t}^{1}\right),
\end{aligned}
$$

with the leading order contributions

$$
\begin{aligned}
& \rho_{r}^{0}=\frac{n-1}{r^{2}}(\sigma-3)[3+(n-1)(3-\sigma)], \\
& p_{r}^{0}=2 \frac{1-n}{r^{2}}(\sigma-3)[2+(n-1)(3-\sigma)], \\
& p_{t}^{0}=\frac{1-n}{r^{2}}(\sigma-3)[2+(n-1)(3-\sigma)],
\end{aligned}
$$

and the post-Newtonian contributions

$$
\begin{aligned}
& \rho_{r}^{1}=2 \rho_{r}^{0}+\frac{(\sigma-3)}{2}+\left(2 \rho_{r}^{0}+\rho_{c}^{1}\right)\left(\frac{r}{r_{c}}\right)^{\sigma}, \\
& p_{r}^{1}=\frac{\sigma-3}{2}+\rho_{c}^{1}\left(\frac{r}{r_{c}}\right)^{\sigma}, \\
& p_{t}^{1}=p_{t}^{0}+\left[p_{t}^{0}+\frac{\sigma(\sigma+1)}{2 n}\right]\left(\frac{r}{r_{c}}\right)^{\sigma},
\end{aligned}
$$


where we have denoted

$$
\rho_{c}^{1}=\frac{1}{2}\left[\frac{\sigma(\sigma+1)}{n}+(1-\sigma)(\sigma-3)\right] .
$$

In what follows, we discuss various energy-conditions, which for an spherically symmetric, static, but anisotropic fluid are subject to the following restrictions:

i) Weak energy conditions: $\rho \geq 0, \rho+p_{r}>0$ and $\rho+p_{t}>0$.

ii) Null energy conditions: $\rho+p_{r} \geq 0$ and $\rho+p_{t} \geq 0$.

iii) Strong energy conditions: $\rho+p_{r} \geq 0, \rho+p_{t} \geq 0$ and $\rho+p_{r}+2 p_{t} \geq 0$.

iv) Dominant energy conditions: $\rho \geq 0, \rho \geq\left|p_{r}\right|$ and $\rho \geq\left|p_{t}\right|$.

In the weak-field approximation the leading order contributions give

$$
\begin{aligned}
\rho_{r}^{0} & =-\frac{(n-1)(3-\sigma)}{r^{2}}[3+(n-1)(3-\sigma)] \leq 0, \\
\rho^{0}+p_{r}^{0} & =\frac{(n-1)(3-\sigma)}{r^{2}}[1+(n-1)(3-\sigma)] \geq 0, \\
\rho^{0}+p_{t}^{0} & =-\frac{(n-1)(3-\sigma)}{r^{2}} \leq 0, \\
\rho^{0}+p_{r}^{0}+2 p_{t}^{0} & =\frac{(n-1)(3-\sigma)}{r^{2}}[5+3(n-1)(3-\sigma)] \geq 0 .
\end{aligned}
$$

The inequalities follow from the conditions $n \geq 1$ and $\sigma \in[0,1)$. Hence to leading order the curvature fluid violates all of the above energy conditions, except for the vacuum GR limit $n=1$. The first order post-Newtonian contributions cannot change this conclusion.

Based on the equivalence of metric $f(R)$ theories with Brans-Dicke metric-scalar field theories with $\omega=0$ [Eqs. (3.97) and (3.101) of Ref. [6]], Ref. [44] has proven that the only spherically symmetric, asymptotically flat vacuum black hole solution for a scalar field with energy-momentum tensor obeying the weak energy condition is the Schwarzschild solution.

The weak-field solution of $f(R)$ theory discussed in this paper leads to an effective energy-momentum tensor that violates all energy conditions. Therefore it can be considered as an approximation of a compact object (for example a black hole) whose parameters fall outside the conditions of the above theorem.

\section{References}

[1] A. G. Riess et al., AJ, 116, 1009 (1998); S. Perlmutter et al., Astrophys. J., 517, 565 (1999); R. A. Knop et al., Astrophys. J., 598, 102 (2003); J. L. Tonry et al., Astrophys. J., 594, 1 (2003); B. J. Barris et al., Astrophys. J., 602, 571 (2004); A. G. Riess et al., Astrophys. J., 607, 665 (2004).

[2] P. de Bernardis et al., Nature, 404, 955 (2000); A. Balbi et al., Astrophys. J., 545, 1 (2000); S. Hanany et al., Astrophys. J., 545, 5 (2000); T. J. Pearson et al., Astrophys. J., 591, 556 (2003).

[3] C. L. Bennett et al., Astrophys. J.S, 148, 1 (2003); D. N. Spergel et al., Astrophys. J.S, 148, 175 (2003).

[4] R. Utiyama, B. S. DeWitt, J. Math. Phys. 3, 608 (1962).

[5] S. Nojiri and S. D. Odintsov, Int. J. Geom. Meth. Mod. Phys. 4, 115 (2007); S. Capozziello and M. Francaviglia, Gen. Rel. Grav. 40, 357 (2008); S. Capozziello, M. De Laurentis, and V. Faraoni, The Open Astr. Jour. 3, 49 (2010); T. P. Sotiriou, V. Faraoni, Rev. Mod. Phys. 82, 451 (2010).

[6] S. Capozziello, V. Faraoni, Beyond Einstein Gravity: A Survey Of Gravitational Theories For Cosmology And Astrophysics (Springer, New York, 2010); S. Capozziello, M. De Laurentis, Invariance Principles and Extended Gravity: Theory and Probes (Nova Science Publishers, New York, 2010).

[7] S. Carloni, P. K. S. Dunsby, S. Capozziello, A. Troisi, Class. Quant. Grav. 22, 4839 (2005); S. Capozziello, V.F. Cardone, A. Trosi, Phys. Rev. D, 71, 043503 (2005).

[8] S. Nojiri, S. D. Odintsov, Phys. Lett. B, 576, 5 (2003); Mod. Phys. Lett. A, 19, 627 (2003); Phys. Rev. D, 68, 12352 (2003); S. M. Carroll, V. Duvvuri, M. Trodden, M. Turner, Phys. Rev. D, 70, 043528 (2004). 
[9] S. Capozziello, Int. J. Mod. Phys. D, 11, 483 (2002).

[10] D. N. Vollick, Phys. Rev. D, 68, 063510 (2003); X. H. Meng, P. Wang, Class. Quant. Grav., 20, 4949 (2003); E.E. Flanagan, Class. Quant. Grav., 21, 417 (2004); X. H. Meng, P. Wang, Class. Quant. Grav., 21, 951 (2004); G.M. Kremer, D. S. M. Alves, Phys. Rev. D, 70, 023503 (2004).

[11] S. Nojiri, S. D. Odintsov, Gen. Rel. Grav., 36, 1765 (2004); X. H. Meng, P. Wang, Phys. Lett. B, 584, 1 (2004).

[12] G. Allemandi, A. Borowiec, M. Francaviglia, Phys. Rev. D, 70, 043524 (2004); 70, 103503 (2004).

[13] V. Faraoni, Cosmology in Scalar-Tensor Gravity (Kluwer Academic Publishers, Dordrecht, 2004).

[14] S. Capozziello, V. F. Cardone, S. Carloni, A. Troisi, Int. J. Mod. Phys. D, 12, 1969 (2003).

[15] S. Capozziello, V. F. Cardone, M. Francaviglia, Gen. Rel. Grav. 38, 711 (2006).

[16] M. Amarzguioui, O. Elgaroy, D. F. Mota, T. Multamaki, Astron. Astrophys. 454, 707 (2006) ; P. Zhang, Phys. Rev. D 73 123504 (2006).

[17] K. S. Stelle, Gen. Rel. Grav., 9, 353 (1978).

[18] I. Quandt, H. J. Schmidt, Astron. Nachr., 312, 97 (1991).

[19] P. D. Mannheim, Astrophys. J., 419, 150 (1993).

[20] S. Capozziello, V. F. Cardone, S. Carloni, A. Troisi, Phys. Lett. A, 326, 292 (2004).

[21] S. Capozziello, M. De Laurentis, S. Nojiri, S. D. Odintsov Gen. Rel. Grav. 41, 2313 (2009).

[22] P. Schneider, J. Ehlers, and E. E. Falco, Gravitational Lenses (Springer - Verlag, Berlin, 1992).

[23] A. O. Petters, H. Levine, J. Wambsganss, Singularity Theory and Gravitational Lensing (Birkhäuser, Boston , 2001).

[24] D. Clowe, M. Bradač, A. H. Gonzalez, M. Markevich, S. W. Randall, C. Jones, and D. Zaritsky, Astrophys. J. 648, L109 (2006).

[25] A. Stabile and A. Stabile, Phys. Rev. D 85, 044014 (2012).

[26] M. Lubini, C. Tortora, J. Näf, Ph. Jetzer, S. Capozziello, Eur. Phys. J. C 71, 1834 (2011).

[27] S. Capozziello, V. F. Cardone, and A. Troisi, Mon. Not. Roy. Astron. Soc. 375, 1423 (2007).

[28] A. Stabile, G. Scelza, Phys. Rev. D 84, 124023 (2011).

[29] G. Magnano, M. Ferraris, and M. Francaviglia, Gen. Rel. Grav. 19465 (1987).

[30] S. Capozziello, M. De Laurentis, M. Francaviglia, S. Mercadante, Found. Phys. 39, 1161 (2009).

[31] T. Biswas, E. Gerwick, T. Koivisto, and A. Mazumdar, Phys. Rev. Lett. 108, 031101 (2012).

[32] S. Capozziello, V. F. Cardone, and A. Troisi, Phys. Rev. D $73104019(2006)$. By replacing $(\alpha, \beta, \xi)$ with $(\delta, \sigma, b)$ we recover the notations of the present paper.

[33] S. Capozziello, A. Stabile, and A. Troisi Phys. Rev. D 76, 104019 (2007).

[34] L. Á. Gergely, T. Harko, M. Dwornik, G. Kupi, and Z. Keresztes, Mon. Not. Roy. Astron. Soc. 415, 3275 (2011).

[35] C. Frigerio Martins and P. Salucci, Mon. Not. Roy. Astron. Soc. 381, 1103 (2007).

[36] Z. Horváth, L. Á. Gergely, and D. Hobill, Class. Quant. Grav. 27, 235006 (2010).

[37] P. Schneider, Astron. Astrophys. 140, 119 (1984); R. Blandford and R. Narayan, Astroph. J. 310, 568 (1986).

[38] K. S. Virbhadra, Phys. Rev. D 79, 083004 (2009).

[39] K. S. Virbhadra and G. F. R. Ellis, Phys. Rev. D 62, 084003 (2000).

[40] M. P. Dábrowski, F. E. Schunck, Astrophys. J., 535, 316 (2000).

[41] W. Jin, I. Platais, and M. A. C Perryman, A Giant Step: From Milli- to MicroArcsecond Astrometry (Cambridge University Press, Cambridge, 2008).

[42] V. Bozza, Phys. Rev. D 78103005 (2008).

[43] C. P. L. Berry and J. R. Gair, Phys. Rev. D 83104022 (2011).

[44] T. P. Sotiriou and V. Faraoni, Phys. Rev. Lett. 108, 081103 (2012). 